\title{
Assessment of anti-saccade parameters to reveal short-term atypical antipsychotic treatment effects in schizophrenia
}

Bilge Çetin İlhan*, Barkın İlhan ${ }^{\dagger}$ and Berna D. Uluğ ${ }^{\ddagger}$

- Konya Training and Research Hospital, Psychiatry Department, Konya, Turkey (blglhn@gmail.com)

- †N.E.Ü Meram Medical Faculty, Biophysics Department, Konya, Turkey $\quad \ddagger$ Hacettepe University Medical Faculty, Biophysics Department, Ankara, Turkey

\section{INTRODUCTION}

In many studies, antipsychotic (AP) treatment effects on schizophrenia have been investigated using certain eye movement paradigms. Among these, the earliest follow-up test is at $\mathbf{6}^{\text {th }}$ week after treatment onset. However, there exist no study so far, focusing on the short-term changes in that period between onset and $6^{\text {th }}$ week.

Accordingly, the primary motivation of our study is to provide a basis to foresee the AP drug efficiency by analyzing the short-term changes on eye movement parameters, far before its real clinical effects.

We present here, the anti-saccade (AS) analysis results from a bigger prospective study covering 24 drug-naïve/washed-out schizophrenia patients vs. healthy controls paired in terms of age, gender and educational status.

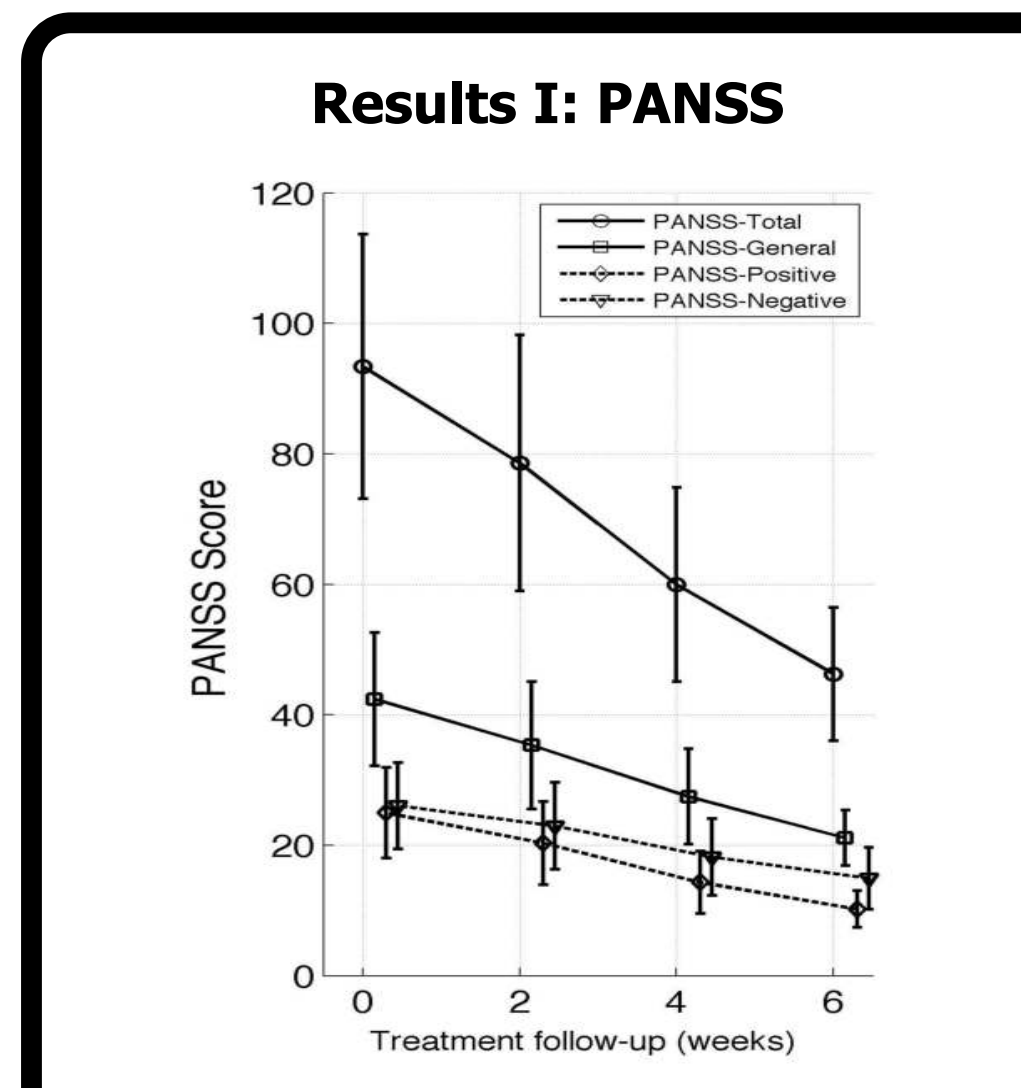

PANSS scores indicate the patients' steady improvement along weeks after the onset of antipsychotic medication.

\section{METHODS}

- ASs to horizontal $20^{\circ}$ or $30^{\circ}$ targets were collected using EOG.

- EOG Experiments and PANSS were held for all 24 schizophrenia patients at $0^{\text {th }}$ (baseline), $2^{\text {nd }}, 4^{\text {th }}$ and $6^{\text {th }}$ weeks of AP treatment, and once for the healthy control group.

-0-phase-shift band-pass and median filtering after baseline correction and calibration. Problematic saccades were excluded via a semi-automated procedure.

- Surrogate data analysis and difference statistics were performed for patients' biweekly experiments against controls to detect for differences in saccadic latency, gain, peak velocity and error rates.

- AS stimuli $\rightarrow$ Saccadic stimuli were presented using a 19" LCD monitor residing at $30 \mathrm{~cm}$ from the plane of the eyes. Subjects' head movements were restrained using a chin-forehead rest. Subjects were asked to fixate over the central target, and execute saccade to the peripheral target which might randomly appear at any of the four possible horizontal locations $\left(20^{\circ}\right.$ and $\mathbf{3 0}^{\circ}$ bilaterally). In total, 25 saccades were recorded associated with each of these four locations.

- Custom Real-time Saccadic/Pursuit Stimulation System $\rightarrow$ Saccadic stimuli were presented using a specially developed hardware/software system (1). The system allows maximum jitter levels lower than 5 us between the actual presentation and the associated trigger of a target stimulus.
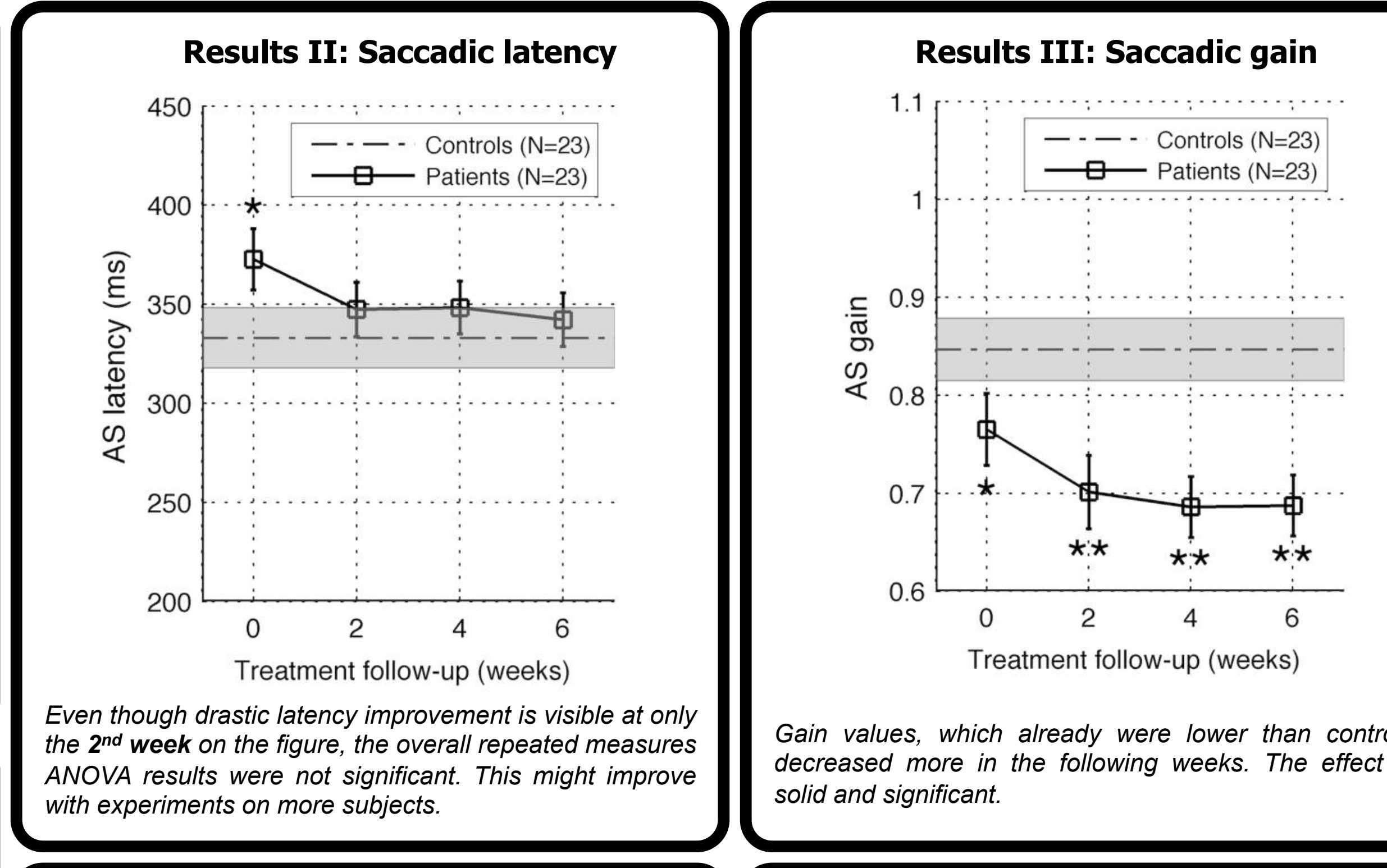

Gain values, which already were lower than controls decreased more in the following weeks. The effect is solid and significant.

Results IV: Peak Velocity

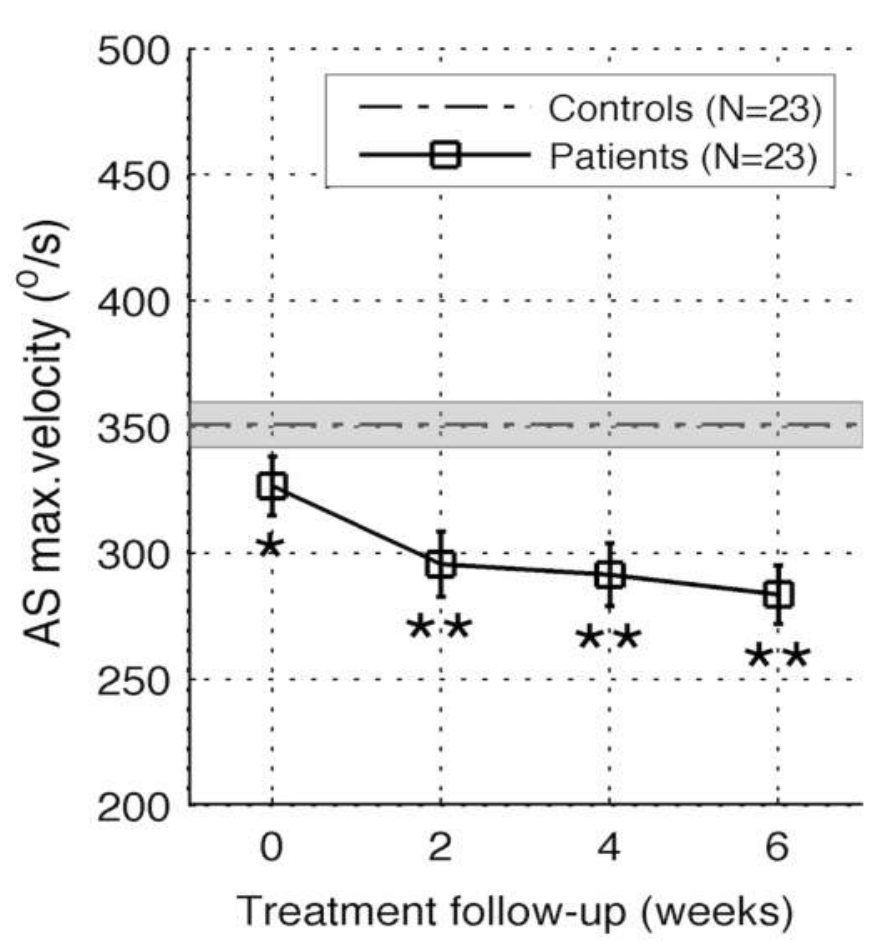

Peak velocity values, which already were lower than controls (the same way as gains) decreased more in the following weeks. This effect is also solid and significant.

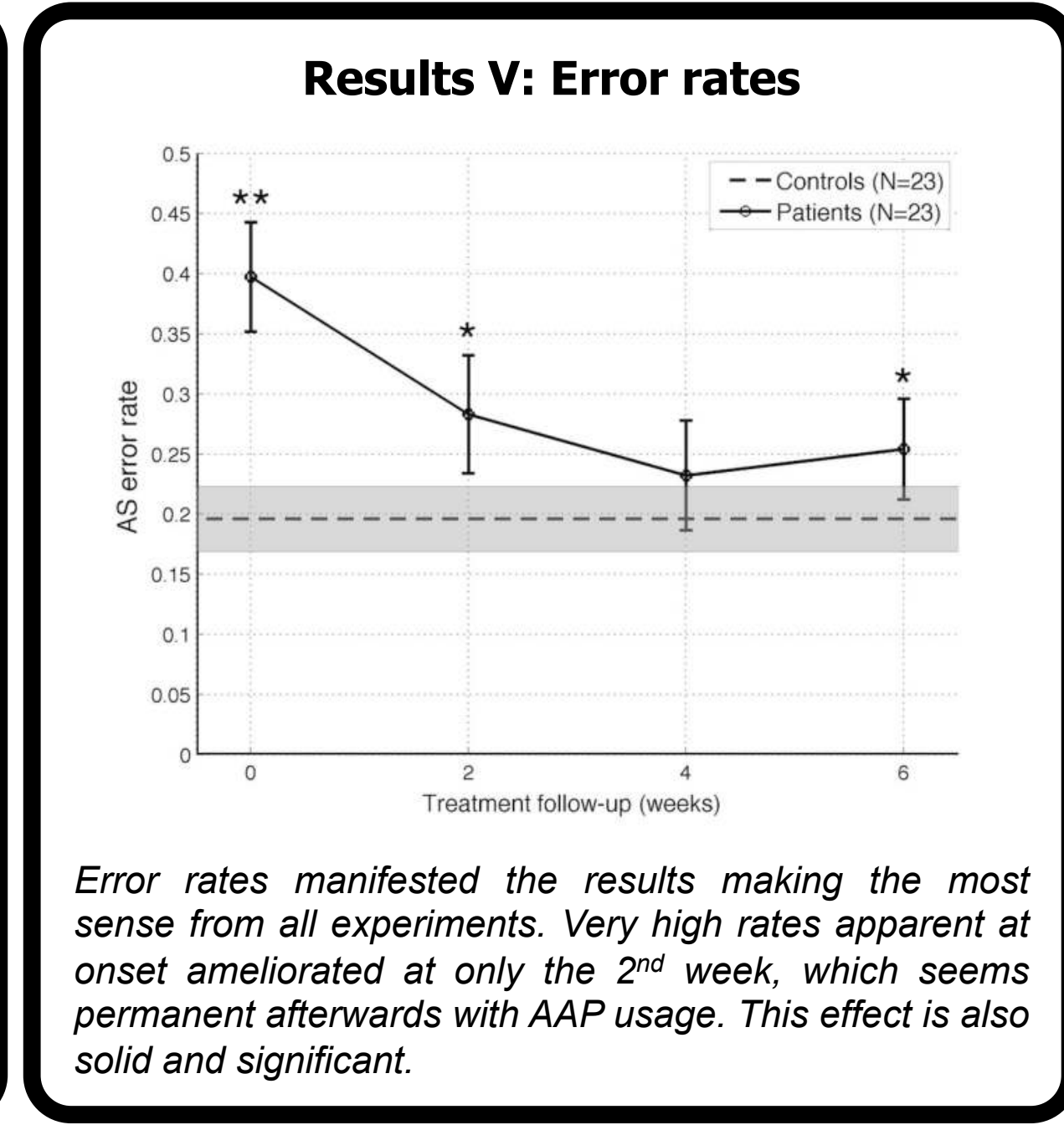

\section{CONCLUSION}

Even though the overall trends for all parameters depict improvement to some degree due to AP treatment, only saccadic gain, peak velocity and saccadic error rates were significant. Saccadic error rate was the most prominent significant parameter was found to decrease steeply at only $2^{\text {nd }}$ week of treatment and continue steadily afterwards. We conclude that new experimental protocols especially focusing on error rates, considering potential correlation with drug effects/side-effects, should be designed in future studies. 